\title{
First Principles Computations of Second-Order Elastic Constants (SOEC) and Equations of State of Rutile $\mathrm{TiO}_{2}$
}

\author{
Ghous Narejo and Warren F. Perger \\ Electrical Engineering Department, Michigan Technological University, 1400 Townsend Drive, Houghton, MI 49931, USA
}

Correspondence should be addressed to Warren F. Perger, wfp@mtu.edu

Received 9 April 2011; Revised 20 June 2011; Accepted 21 June 2011

Academic Editor: Ravindra R. Pandey

Copyright ( $) 2011$ G. Narejo and W. F. Perger. This is an open access article distributed under the Creative Commons Attribution License, which permits unrestricted use, distribution, and reproduction in any medium, provided the original work is properly cited.

First principles computations of second-order elastic constants (SOECs) and bulk moduli $(B)$ are carried out by ELASTCON and equation of state (EOS) programs. Computational results of lattice parameters, elastic constants, and bulk moduli are obtained with a wide variety of potentials and a limited combination of basis sets and are compared with computational and experimental results by other researchers in the field. DFT hybrid potentials provided the best comparison with the experiment.

\section{Introduction}

Titanium dioxide $\left(\mathrm{TiO}_{2}\right)$ is an important transition metal oxide, which exists in anatase, rutile, and brookite phases [1]. There is a considerable interest in the fabrication [2] of this material. It is employed extensively in semiconductors, optical devices, photovoltaic cells, gas sensors, and electrochemical storage devices [3]. Due to its technological importance, the crystalline structure and properties of rutile $\mathrm{TiO}_{2}$ phases have remained a major focus of researchers $[1,4,5]$.

$A b$-initio Hartree Fock (HF) and density functional theory (DFT) techniques have been employed by various research groups to compute the optimized electronic structure, band gap, and charge density of rutile $\mathrm{TiO}_{2}[1,6-8]$.

The ab-initio computation of the elastic constants and bulk moduli of rutile $\mathrm{TiO}_{2}$ is presented in this work using the CRYSTAL09 code [9]. The availability of precise and accurate values of experimental lattice parameters, elastic properties, [10] and chemical bonding [11] has provided a considerable challenge to ab-initio computational codes. Computational codes based upon the linear combination of atomic orbitals (LCAO) and plane waves (PW) were employed to compute the optimized crystalline structure of rutile $\mathrm{TiO}_{2}[1,6-8][5]$. However, the inadequacy of the existing potentials $[1,6,7]$ to accurately predict the physics of $\mathrm{TiO}_{2}$ has motivated a detailed analysis of a wide range of existing and new potentials $[6,12]$. Research on the structural and electronic properties has confirmed the relative accuracy of the hybrid potentials $[1,6,7,12]$.

There has been no systematic effort to explore the elastic properties of rutile $\mathrm{TiO}_{2}$ using the LCAO code CRYSTAL09 [9]. CRYSTAL09 possesses a combination of geometry optimization techniques, basis sets, potentials, and algorithms such as ELASTCON [9] (for second-order elastic constants) and EOS (equation of state) [9]. The term ELASTCON represents a computational algorithm that calculates second-order elastic constants and bulk moduli in a highly automated manner. The algorithm possesses various parameters which can be explored to enhance the computational accuracy for a wide variety of crystalline systems. The experimental values of lattice parameters, elastic constants and bulk moduli [10] of the $\mathrm{TiO}_{2}$ rutile phase provided an opportunity to confirm the relative merit of the self-consistent field (SCF) process, the ELASTCON, and EOS programs $[13,14]$.

\section{Potentials and Basis Sets}

The HF, local and non-local DFT potentials have consistently resulted in inadequate results of the lattice parameters. First principles computations using PW codes [15-20] can not employ the hybrid mixing of HF exchange and DFT correlations.

Therefore, we employed hybrid potentials in our computations of elastic properties as suggested in $[1,6,7]$. The 
hybridization between the $\mathrm{Ti} d$-orbitals and $\mathrm{O} p$-orbitals requires that an adequate percentage of exchange and correlation is introduced in the potential while computing the electronic structure of a material [21]. The DFT-PWGGA and DFT-PBE potentials lack the exchange part and HF lacks the correlation part, barring them from being as accurate as hybrid potentials.

In addition to using hybrid potentials, the proper choice of basis sets, SCF tolerances, ELASTCON, and EOS parameters can achieve the optimum efficiency as well as accuracy (see Sections 5 and 7). Moreover, it is necessary to employ adequate SCF tolerances consistently.

We selected the O-8411d1 [22] and O-6311d1 [23] basis sets for $\mathrm{O}$ atoms and defined these basis sets as basis set 1 and 2, respectively. Both basis sets used a pseudopotential basis set for the Ti atom [9]. The aim of employing these combinations was to separate the basis set dependency from other factors such as potentials, SCF parameters, ELASTCON and EOS tolerances.

The sensitivity of the elastic properties with respect to deviations in the lattice parameters [1] suggests that a wellchosen HF, DFT, and hybrid potentials must be employed to compute the lattice parameters and understand their influences on the elastic constant values. The impact of a particular potential and basis set on the computational values of lattice parameters, elastic constants, and bulk moduli values can be understood by careful comparisons of results achieved for a variety of these basis sets and potentials.

Further, we have also compared our computational results for each potential and basis set combination with $[1,7]$ to confirm their findings.

\section{Computational Procedure}

We employed two different basis sets and a variety of potentials to compute the optimized lattice constants and elastic properties (see Section 2 for the discussion of potentials and basis sets). The ELASTCON [9] and EOS [9] algorithms are employed to compute the elastic constants and bulk moduli of rutile $\mathrm{TiO}_{2}$ in an automated manner.

The two basis sets employed are named as basis set 1 and 2 (see Section 2 for basis set definitions). Optimized lattice parameters are computed with HF, DFT, and hybrid potentials using the two unique basis sets. The computations of elastic constants and bulk moduli are performed by employing the ELASTCON algorithm [9]. The computations of the equation of state and bulk moduli are carried out by the EOS algorithm. Finally, the bulk moduli obtained with ELASTCON and EOS algorithms are compared.

Optimized lattice parameters, elastic constants, and bulk moduli, obtained with basis sets 1 and 2, are also compared with the experimental values where possible. A possible contribution of this research work is to assist a general reader in understanding the complex dependance of the elastic properties on the quality of basis sets, potentials, SCF process, ELASTCON, and EOS parameters. Experimental values of lattice parameters, elastic constants, and bulk moduli of rutile $\mathrm{TiO}_{2}$ provide additional assistance for implementation of ELASTCON and EOS programs.
A significant number of computations and experiments have been conducted on rutile $\mathrm{TiO}_{2}$. It is also noticed that a high precision has been achieved in the experimental lattice parameters of rutile $\mathrm{TiO}_{2}$ [24]. The availability of the experimental values of lattice constants, elastic constants, and bulk moduli of rutile $\mathrm{TiO}_{2}$ provides a highly valuable resource to conduct new research. In contrast, computations of elastic constants and bulk moduli have been obtained in an isolated and nonsystematic manner.

A vast majority of ab-initio computations have already tried DFT exchange and correlation potentials $[1,7]$. The need for hybrid potentials arose because HF underestimated and DFT potentials overestimated the lattice parameters of rutile $\mathrm{TiO}_{2}$. It has been found that the higher exchange and correlation associated with the transition metals requires new hybrid potentials with variable exchange and correlation $[1,7]$. The HF, DFT, and hybrid potentials are employed in this work [9] to fill the gap with regard to the efficient, accurate, and systematic computation of elastic properties of rutile $\mathrm{TiO}_{2}$.

\section{Computational Algorithms}

4.1. ELASTCON. The computation of elastic constants and bulk moduli is an automated procedure within the ELASTCON algorithm. The computational process begins with determining the crystalline symmetry of rutile $\mathrm{TiO}_{2}$. The analytic first derivative and the numerical second derivative of the total energy are carried out for each specific strain. Levenberg Marquardt (LM) curve fitting [25] is used to compute the required elastic constants for the rutile $\mathrm{TiO}_{2}$ symmetry.

The elastic constants can be extracted from the second derivative of the total energy as

$$
C_{\alpha \beta}=\left.\frac{1}{V} \frac{\partial^{2} E}{\partial \epsilon_{\alpha} \partial \epsilon_{\alpha}}\right|_{0} .
$$

In (1), the terms $C_{\alpha \beta}, E$, and $V$ express the elastic constant tensor, energy, and volume of the crystalline structure, respectively.

The appropriate number of strains is applied in a systematic manner, the elastic constants are calculated, and the compliance coefficients are computed from (3). The compliance coefficients are then utilized for the computation of the bulk modulus as shown in the following:

$$
\begin{gathered}
B=\frac{1}{S_{11}+S_{22}+S_{33}+2\left(S_{12}+S_{13}+S_{23}\right)}, \\
{[S]=[C]^{-1} .}
\end{gathered}
$$

The terms $S_{i j}$ and $B$ in (2) express the compliance tensor elements and bulk modulus, respectively. Whereas the term $C$ represents the second-order elastic constant.

4.2. EOS. The EOS algorithm [13] employs changes in the optimized volume of rutile $\mathrm{TiO}_{2}$ in a systematic manner. Additionally, the EOS algorithm allows the user to select a range of volumes and number of volumes within that 
range. For each of the volumes in the range, the CRYSTAL09 optimizes the internal coordinates and lattice parameters while keeping the volume constant. The energy versus volume results are then curve-fitted to an EOS such as the Murnaghan EOS [26] shown in (4) (see [13] for the CVOLOPT option employed during the constant volume geometry optimization for EOS computations).

The EOS algorithm in CRYSTAL09 contains a diversity of equations of state such as Birch Murnaghan, third-order Birch Murnaghan, logarithmic, Vinet, and polynomial. The third-order Birch Murnaghan equation of state algorithm utilized for computing the bulk moduli from the energy versus volume computations for rutile $\mathrm{TiO}_{2}$ is

$$
\begin{aligned}
E(V)=B_{0} V_{0}[ & \frac{1}{B^{\prime}\left(B^{\prime}-1\right)}\left(\frac{V_{0}}{V}\right)^{B^{\prime}-1} \\
& \left.+\frac{V}{B^{\prime} V_{0}}-\frac{1}{B^{\prime}-1}\right]+E_{0} .
\end{aligned}
$$

In (4), $V_{0}$ represents the volume at the minimum energy, $B_{0}$ is the bulk modulus at pressure $P=0, B^{\prime}$ is the derivative of the bulk modulus at $P=0$, and $E_{0}$ is the minimum energy.

The bulk moduli results are obtained with LevenbergMarquardt curve fitting of the $E$ versus $V$ computations. The detailed discussion about the ELASTCON [9] and EOS [9] algorithms can be seen in $[13,14]$.

\section{Computational versus Experimental Attempts}

Due to its technological importance, a significant number of experiments have been conducted on rutile $\mathrm{TiO}_{2}[10,20,27]$. The experimental results of the electronic structure, band gap, and optic and elastic properties are available for rutile $\mathrm{TiO}_{2}$. The lattice parameters of the rutile $\mathrm{TiO}_{2}$ have been determined [24] precisely up to the fifth significant figure. In addition, the pressure and temperature dependence of elastic constants and bulk moduli have also been explored through experimental means.

The experimental and computational values of elastic constants and bulk moduli [10] of rutile $\mathrm{TiO}_{2}$ have special significance as they serve the purpose of linking these vital branches of research. The importance of achieving a consistency in the lattice parameters of rutile $\mathrm{TiO}_{2}$ among diverse experimental techniques and computational codes is a remarkable achievement which has been repeatedly observed $[1,6,7]$.

However, there are deviations in the experimental values of elastic constants and bulk moduli due to their dependence on the experimental details, pressure, and temperature conditions [28]. Therefore, the sources that cause the variations in the experimental values $[10,28-30]$ must also be considered. However, the dependence of the experimental results on the nature of the experimental setup and ambient conditions can be explored and understood by robust and reliable ab-initio computational methods.

An increase in pressure has shown an increase in the elastic constants and bulk moduli values [31] for rutile $\mathrm{TiO}_{2}$. Moreover, the values of $C_{11}, C_{33}, C_{66}, C_{12}$, and $C_{13}$ increase
TABLE 1: The values of relaxed lattice constants (in $\AA$ ), ambient volume (in $\AA^{3}$ ), and total energy, $E$ (in a.u.), for rutile $\mathrm{TiO}_{2}$. The computations used Hartree-Fock, DFT LDA, PWGGA, BLYP, B3LYP, and B3PW potentials using basis sets 1 and 2 (even numbered rows represent computational values obtained with basis set 2). The numbers within parentheses are percent differences from experiment. (See Sections 2, 3, 5, and 7).

\begin{tabular}{lccc}
\hline & $a(\AA)$ & $c(\AA)$ & Vol. $\left(\AA^{3}\right)$ \\
\hline HF & $4.568(-.54)$ & $2.980(.74)$ & $62.11(0)$ \\
& $4.561(-.70)$ & $2.991(1.10)$ & $62.24(0)$ \\
LDA & $4.559(.74)$ & $2.932(.87)$ & $60.98(2)$ \\
& $4.539(-1.18)$ & $2.904(-1.85)$ & $59.84(4)$ \\
PWGGA & $4.640(1)$ & $2.976(.33)$ & $64.08(2)$ \\
& $4.619(.56)$ & $2.946(-.40)$ & $62.82(1)$ \\
PBE & $4.647(1.17)$ & $2.978(.67)$ & $64.32(3)$ \\
& $4.625(.69)$ & $2.949(-.30)$ & $63.05(1.03)$ \\
BLYP & $4.66(1.45)$ & $3.01(1.75)$ & $65.67(5)$ \\
& $4.657(1.37)$ & $2.971(.43)$ & $64.41(3.12)$ \\
B3LYP & $4.629(.78)$ & $2.976(.60)$ & $63.78(2)$ \\
& $4.607(.21)$ & $2.957(-.03)$ & $62.75(.55)$ \\
B3PW & $4.599(.13)$ & $2.961(.10)$ & $62.63(0)$ \\
& $4.583(-.21)$ & $2.942(-.54)$ & $61.81(.95)$ \\
PBE0 & $4.627(.73)$ & $2.973(.50)$ & $63.69(2)$ \\
& $4.571(-.48)$ & $2.940(-.61)$ & $61.46(1.52)$ \\
Exps. $[2,4,10,32]$ & 4.593 & 2.958 & 62.40 \\
\hline
\end{tabular}

with increasing pressure, whereas the pressure dependence of $C_{44}$ is not clear. Moreover, the elastic constants are nonlinearly dependent on the temperature.

Another important area where experiments were performed is the volume charge density and chemical bonding of rutile $\mathrm{TiO}_{2}$ [11]. The quantitative convergent beam electron diffraction (QCBED) technique was employed to determine the experimental volume charge density and chemical bonding. The experimental data was utilized to confirm the contribution of ionic and covalent bonding in rutile $\mathrm{TiO}_{2}$. The experimental charge density maps predicted the $p$ - $d$ hybridization between Ti 3- $d$ electrons and $\mathrm{O}$ ligands.

The charge density influenced by highly localized $d$ orbitals of Ti atoms impacts the selection of proper basis sets and potentials in ab-initio computations (see Sections 2 and 7).

Unlike the precision in the experimental lattice parameters, the computational values of lattice parameters vary in the second significant figures. The variation in the lattice parameter values is partially due to the complex nature of the chemical bonding of rutile $\mathrm{TiO}_{2}$. Moreover, the sensitivity of the computational values of lattice parameters is attributed to the choice of potentials and basis sets. However, the hybrid potentials can map the chemical bonding and charge density of rutile $\mathrm{TiO}_{2}$ with a considerable accuracy.

In general, the ab-initio computations are lacking the level of accuracy of the experiments. However, the extensive computational effort by $[1,6,7,34]$ has provided guidance for the present study. 
TABLE 2: The elastic constants and bulk modulus computational results using the Hartree Fock and DFT LDA, PWGGA, BLYP, B3LYP, and B3PW potentials with basis set 1 . All values are in GPa. The numbers within parentheses are percent differences from experiment. (See Sections 2, 3, 5, and 7).

\begin{tabular}{|c|c|c|c|c|c|c|c|}
\hline & $C_{11}$ & $C_{12}$ & $C_{13}$ & $C_{33}$ & $C_{44}$ & $C_{66}$ & $B$ \\
\hline $\mathrm{HF}$ & 364. (36) & 216. (23) & 184. (25) & 626. (29) & 164. (32) & 277. (456) & $270 .(27,17)$ \\
\hline LDA & 311. (16) & 211. (21) & 175. (4) & 504. (28) & 159. (33) & 253. (15) & 243. $(15,6)$ \\
\hline PWGGA & 266. $(-1)$ & 177. (1) & 149. (1) & 462. $(-5)$ & 138. (11) & 223. (17) & 208. $(-2,-10)$ \\
\hline $\mathrm{PBE}$ & 262. $(-2)$ & 176. (1) & 146. $(-1)$ & 458. $(-6)$ & 135. (9) & 221. (16) & 205. $(-3,-11)$ \\
\hline BLYP & 256. $(-5)$ & 133. $(-24)$ & 143. (3) & 478. $(-1)$ & 154. $(-19)$ & 206. (8) & 188. $(-11,-18)$ \\
\hline B3LYP & 281. (5) & 186. (6) & 157. (7) & 506. (5) & 140. (13) & 236. (24) & 220. $(4,-4)$ \\
\hline B3PW & 294. (10) & 194. (11) & 164. (12) & 517. (7) & 147. (19) & 246. (29) & 229. $(8,-1)$ \\
\hline PBE0 & 277. (3) & 185. (6) & 155. (5) & 494. (4) & 139. (12) & 234. (23) & 217. $(2,-6)$ \\
\hline Exps. $[10,29,30,33]$ & 268.00 & 175.00 & 147.00 & 484.00 & 124.00 & 190.00 & $212.00,230.00$ \\
\hline
\end{tabular}

TABLE 3: The elastic constants and bulk modulus computational results using the Hartree Fock and DFT LDA, PWGGA, BLYP, B3LYP, and B3PW potentials with basis set 2. All values are in GPA. The numbers within parentheses are percent differences from experiment. (See Sections 2, 3, 5, and 7).

\begin{tabular}{|c|c|c|c|c|c|c|c|}
\hline & $C_{11}$ & $C_{12}$ & $C_{13}$ & $C_{33}$ & $C_{44}$ & $C_{66}$ & $B$ \\
\hline $\mathrm{HF}$ & 393. (47) & 237. (35) & $210 .(43)$ & 662. (37) & 171. (38) & 303. (60) & 295. $(39,28)$ \\
\hline LDA & 171. $(-36)$ & 385. (120) & 205. (39) & 577. (19) & 130. (5) & 279. (47) & 266. $(23,16)$ \\
\hline PWGGA & 223. $(-17)$ & 273. (56) & 182. (24) & 522. (8) & 123. $(-1)$ & 246. (29) & 237. $(12,3)$ \\
\hline PBE & $220 .(-18)$ & 270. (54) & 181. (23) & 517. (7) & 122. $(-2)$ & 244. (28) & 235. $(11,2)$ \\
\hline BLYP & 260. $(-3)$ & 226. (29) & 175. (19) & 509. (5) & 123. $(-1)$ & 236. (24) & 231. $(9,1)$ \\
\hline B3LYP & 295. (10) & 240. (37) & 193. (31) & 562. (16) & 137. (10) & 261. (37) & 255. $(20,11)$ \\
\hline B3PW & 269. (0) & 269. (54) & 197. (34) & 569. (18) & 137. (10) & 267. (41) & 257. $(21,12)$ \\
\hline PBE0 & 267. $(-1)$ & 282. (61) & 203. (38) & 579. (20) & 139. (12) & 273. (44) & 263. $(24,14)$ \\
\hline Exps. $[10,29,30,33]$ & 268.00 & 175.00 & 147.00 & 484.00 & 124.00 & 190.00 & $212.00,230.00$ \\
\hline
\end{tabular}

\section{Computational Parameters}

For the computation of lattice parameters and elastic properties, the SCF tolerances and other computational parameters were carefully chosen. The ELASTCON, EOS, and SCF tolerances were adjusted due to the highly localized nature of transition metal Ti $d$-orbitals. The ELASTCON and other parameters were chosen as STEPSIZE $=0.01$, NUMDERIV $=7$, LGRID $=(75,434)$, and SHRINK $=9 \times 9$. The SCF tolerances were fixed as TOLINTEG $=999918$ and TOLDEE $=9[20]$.

\section{Discussion of Results}

Table 1 shows the optimized lattice parameters computed with basis sets 1 and 2, respectively. The unit cell of rutile $\mathrm{TiO}_{2}$, computed with CRYSTAL09 code, is shown in Figure 1. The percentage deviation between the computational and experimental values of lattice parameters and volume is also shown for basis sets 1 and 2 . The experimental values from $[2,4,10]$ are provided at the bottom of Table 1 . Agreement between the computational and experimental values of lattice parameters can be observed for basis sets 1 as well as 2 .

Elastic constants and bulk moduli computed with ELASTCON are presented in Tables 2 and 3. Significant

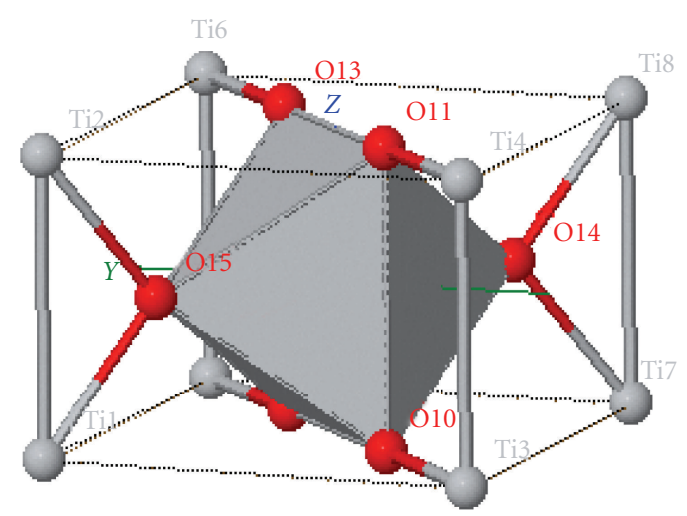

Figure 1: The rutile $\mathrm{TiO}_{2}$ crystalline structure is shown. Ti and $\mathrm{O}$ atoms are shown as grey and red spheres. The distorted octahedral is formed between $\mathrm{Ti}$ and $\mathrm{O}$ atoms.

agreement between the computational and experimental values of elastic constants and bulk moduli is found for basis sets 1 and 2 which can be observed in Figures 2 and 3 . The percentage deviation between the computational and experimental values of elastic constants and bulk moduli is shown in Tables 2 and 3. 
TABLE 4: Equation of state results for rutile $\mathrm{TiO}_{2}$ with the Birch Murnaghan third-order equation. The energy-volume curve was fitted with eleven points, and the range of volume around equilibrium was chosen as $\pm 10 \%$ using basis sets 1 and 2 (computational results with basis 2 shown in even-numbered rows). (See Sections 2, 3, 5, and 7). The value of the bulk modulus, $B_{\mathrm{EL}}$, calculated separately, is given in the last column for comparison.

\begin{tabular}{lccc}
\hline & $B_{\mathrm{EOS}}(\mathrm{GPa})$ & $V_{o}\left(\AA^{3}\right)$ & $B_{\mathrm{EL}}(\mathrm{GPa})$ \\
\hline HF & 267. & 62.29 & 270. \\
\multirow{2}{*}{ LDA } & 293. & 62.31 & 295. \\
\multirow{2}{*}{ PWGGA } & 241. & 60.98 & 243. \\
& 279. & 59.84 & 283. \\
PBE & 206. & 64.09 & 208. \\
& 239. & 62.87 & 237. \\
BLYP & 202. & 64.33 & 205. \\
& 236. & 63.10 & 235. \\
B3LYP & 191. & 65.93 & 188. \\
& 229. & 64.45 & 231. \\
B3PW & 217. & 63.81 & 220. \\
& 252. & 62.81 & 255. \\
PBE0 & 226. & 62.66 & 229. \\
& 256. & 61.83 & 257. \\
Exps. $[10,29,30,33]$ & - & 62.24 & 217.12 \\
\hline
\end{tabular}

Table 4 and Figure 3 show the comparisons between the bulk moduli values computed with ELASTCON and EOS algorithms. Excellent agreement is observed between the computational values of bulk moduli achieved with ELASTCON and EOS programs. The agreement between the bulk moduli values points at the computational accuracy of the ELASTCON and EOS programs. Moreover, it is important that the computational results should also be crosschecked with the experimental values of the bulk moduli given at the bottom of Table 4.

However, there is a considerable disagreement between the computational and experimental values of the elastic constants and $B$ for basis set 1 and 2 with HF and DFTLDA potentials as shown in Tables 2 and 3. On the other hand, the computational values of the elastic constants and $B$ provide a significant agreement with the experimental values computed with DFT-PWGGA, DFT-PBE, and hybrid potentials for basis set 1 as shown in Table 2 .

The hybrid potentials have shown better agreement due to the adequate percentage of exchange and correlation contributions to total energy of the crystal specifically important for the highly correlated physics of the Ti transition metal. The localized nature of the Ti atom $d$-orbitals contributes to the higher exchange and correlation effects.

The HF, local DFT, and nonlocal DFT potentials cannot predict results as effectively as hybrid potentials. It can be easily seen in Tables 1-4. In fact, the lack of correlation in HF and the localized nature of DFT-LDA potentials make these potentials less effective for transition metal oxides which

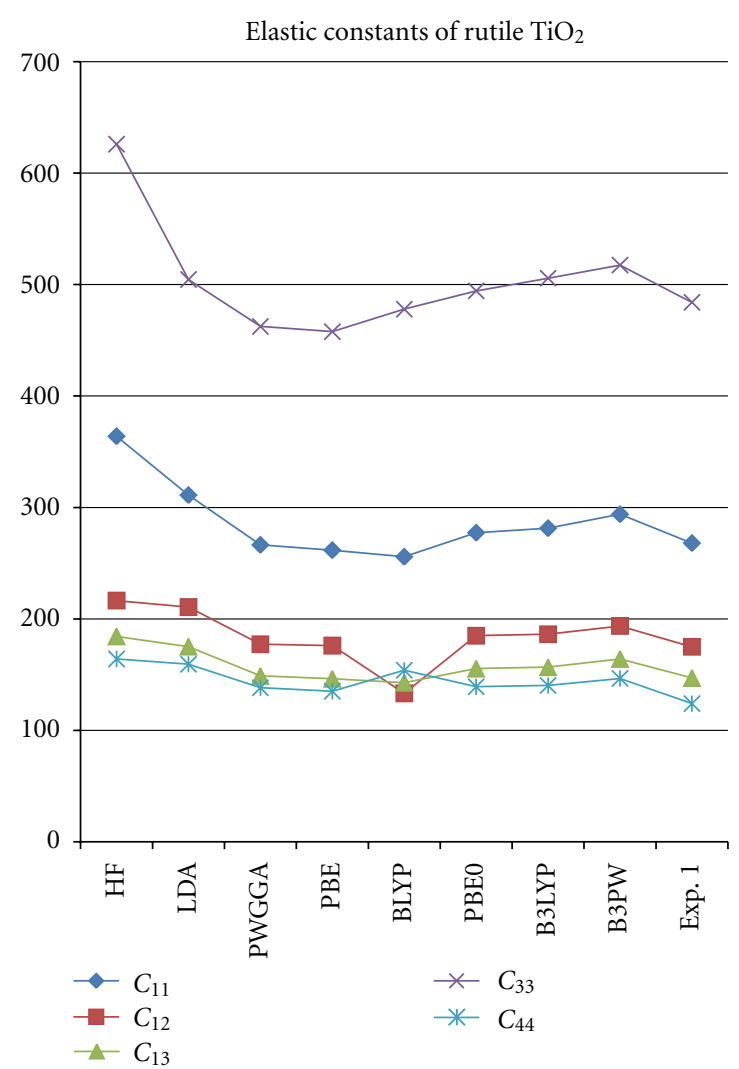

FIGURE 2: The computational and experimental values of elastic constants and bulk moduli are shown. All values are in GPa.

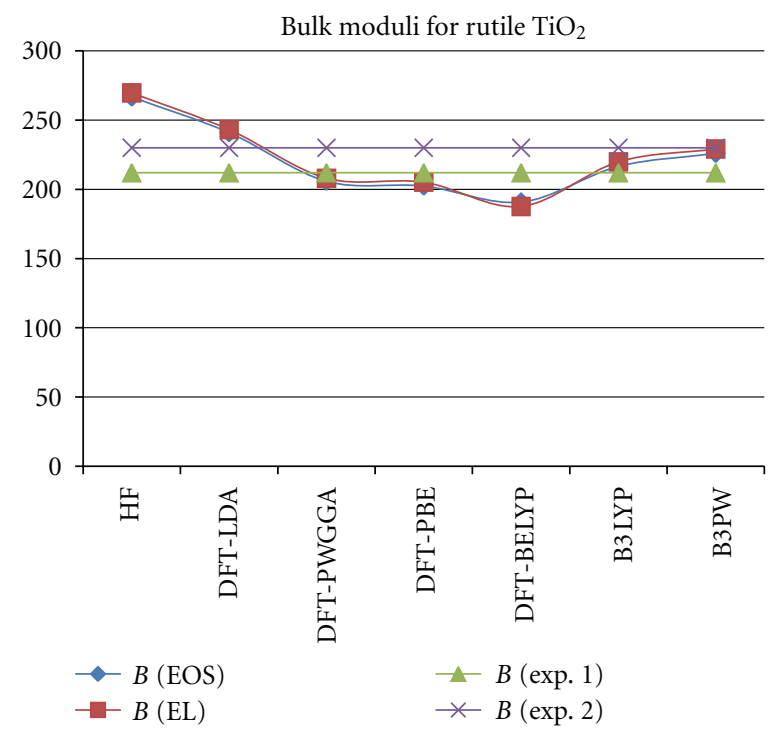

FIGURE 3: The computational and experimental values of bulk moduli are shown. All values are in GPa.

possess the covalent as well as ionic nature of chemical bonding.

It must be mentioned that DFT-PWGGA and DFT-PBE exhibit better agreement with experimental results due to the nonlocalized nature of the rutile $\mathrm{TiO}_{2}$ volume charge density. However, the agreement between the DFT-PWGGA, 
DFT-PBE, and experimental results of elastic constants may not be adequate which can be confirmed by values in Tables 1,3 , and 4 .

\section{Conclusions}

The technological applications of titanium dioxide $\left(\mathrm{TiO}_{2}\right)$ have generated a significant research activity in experimental and computational sciences. Computational research has resulted in testing the merit of basis sets, potentials, and new programs. The employment of basis sets 1 and 2 has resulted in a considerable agreement between the computational and experimental values of lattice parameters, elastic constants, and bulk moduli. The elastic properties of rutile $\mathrm{TiO}_{2}$ are computed and compared with experimental values. The dependance of experimental values on experimental setup, temperature, and pressure conditions cannot be ignored. The computations of elastic constants and bulk moduli by a wide variety of $a b$-initio techniques provide results to generate new experiments on this material.

We have separated the factors that determine the quality of computational results of the lattice parameters and elastic properties. The nonlocal DFT and hybrid potentials present better agreement with the experimental values of lattice parameters, elastic constants, and bulk moduli. However, the disagreement between the computational and experimental values of the elastic constants and bulk moduli for $\mathrm{HF}$ and DFT-LDA potentials $[15,16,18]$ is significant.

The results presented for rutile $\mathrm{TiO}_{2}$ with different potentials are motivated by a variety of challenges related to the existing potentials. For a crystalline system such as rutile $\mathrm{TiO}_{2}$, which has considerable visibility in the experimental research arena, an integrated set of computational results have significant utility. These computations can guide researchers to appreciate the subtle influences of the charge density on lattice parameters and elastic properties of rutile $\mathrm{TiO}_{2}$.

\section{Acknowledgment}

One of the authors (W. F. Perger) gratefully acknowledges the support of the Office of Naval Research Grant N00014-01-10802 through the MURI program.

\section{References}

[1] F. Labat, P. Baranek, C. Domain, C. Minot, and C. Adamo, "Density functional theory analysis of the structural and electronic properties of $\mathrm{TiO}_{2}$ rutile and anatase polytypes: performances of different exchange-correlation functionals," The Journal of Chemical Physics, vol. 126, no. 15, pp. 1-12, 2007.

[2] S. Tanemuraa, L. Miaoa, W. Wunderlicha et al., "Fabrication and characterization of anatase/rutile- $-\mathrm{TiO}_{2}$ thin films by magnetron sputtering: a review ", Science and Technology of Advanced Materials, vol. 6, no. 1, pp. 11-17, 2005.

[3] S. D. Mo and W. Y. Ching, "Electronic and optical properties of three phases of titanium dioxide: rutile, anatase, and brookite," Physical Review B, vol. 51, no. 19, pp. 13023-13032, 1995.

[4] H. Yao, L. Ouyang, and W. Y. Ching, "Ab initio calculation of elastic constants of ceramic crystals," Journal of the American Ceramic Society, vol. 90, no. 10, pp. 3194-3204, 2007.
[5] D. I. Bilc, R. Orlando, R. Shaltaf, G. M. Rignanese, J. Íñiguez, and P. Ghosez, "Hybrid exchange-correlation functional for accurate prediction of the electronic and structural properties of ferroelectric oxides," Physical Review B, vol. 77, article 165107, no. 16, pp. 1-13, 2008.

[6] F. Corá, "The performance of hybrid density functionals in solid state chemistry: the case of $\mathrm{BaTiO}_{3}$," Molecular Physics, vol. 103, no. 18, pp. 2483-2496, 2005.

[7] C. Adamo and V. Barone, "Toward reliable density functional methods without adjustable parameters: the PBE0 model," Journal of Chemical Physics, vol. 110, no. 13, pp. 6158-6170, 1999.

[8] M. E. Stizaumainis, T. Ejima, and W. J. James, "The $\mathrm{TiO}_{2}$ phase explored by the lattice constant and density method," Acta Crystallographica, vol. 8, pp. 493-497, 1961.

[9] R. Dovesi, V. R. Saunders, C. Roetti et al., CRYSTAL09 User's Manual, University of Torino, Torino, Italy, 2009.

[10] M. Iuga, G. Steinle-Neumann, and J. Meinhardt, "Ab-initio simulation of elastic constants for some ceramic materials," European Physical Journal B, vol. 58, no. 2, pp. 127-133, 2007.

[11] B. Jiang, J. M. Zuo, N. Jiang, M. O’Keeffe, and J. C. H. Spence, "Charge density and chemical bonding in rutile, $\mathrm{TiO}_{2}$," Acta Crystallographica, vol. 59, no. 4, pp. 341-350, 2003.

[12] F. Corá, M. Alfredsson, G. Mallia et al., Principles and Applications of Density Functional Theory in Inorganic Chemistry II, 2004.

[13] W. F. Perger, J. Criswell, B. Civalleri, and R. Dovesi, "Ab-initio calculation of elastic constants of crystalline systems with the CRYSTAL code," Computer Physics Communications, vol. 180, no. 10, pp. 1753-1759, 2009.

[14] W. F. Perger, "First-principles calculation of second-order elastic constants and equations of state for lithium azide, $\mathrm{LiN}_{3}$, and lead azide, $\mathrm{Pb}\left(\mathrm{N}_{3}\right)_{2}$," International Journal of Quantum Chemistry, vol. 110, no. 10, pp. 1916-1922, 2010.

[15] M. Mikami, S. Nakamura, O. Kitao, H. Arakawa, and X. Gonze, "First-principles study of titanium dioxide: rutile and anatase," Japanese Journal of Applied Physics, vol. 39, no. 8 B, pp. L847-L850, 2000.

[16] X. Ma, P. Liang, L. Miao et al., "Pressure-induced phase transition and elastic properties of $\mathrm{TiO}_{2}$ polymorphs," Physica Status Solidi (B), vol. 246, no. 9, pp. 2132-2139, 2009.

[17] Y. Liu, L. Ni, Z. Ren, G. Xu, C. Song, and G. Han, "Negative pressure induced ferroelectric phase transition in rutile $\mathrm{TiO}_{2}$," Journal of Physics Condensed Matter, vol. 21, article 275901, no. 27, 2009.

[18] J. Muscat, V. Swamy, and N. M. Harrison, "First-principles calculations of the phase stability of $\mathrm{TiO}_{2}$," Physical Review B, vol. 65, no. 22, Article ID 224112, pp. 2241121-22411215, 2002.

[19] E. Shojaee and M. R. Mohammadizadeh, "First-principles elastic and thermal properties of $\mathrm{TiO}_{2}$ : a phonon approach," Journal of Physics Condensed Matter, vol. 22, no. 1, pp. 1-8, 2010.

[20] J.-Z. Zhao, G.-T. Wang, and Y.-C. Liang, "Mechanical properties and electronic structures of cotunnite $\mathrm{TiO}_{2}$," Chinese Physics Letters, vol. 25, no. 12, pp. 4356-4359, 2008.

[21] G. Francois and K. Winfried, "Lattice dynamics of oxides with rutile structure and instabilities at the metal-semiconductor phase transitions of $\mathrm{NbO}_{2}$ and $\mathrm{VO}_{2}, "$ Physical Review B, vol. 31, no. 8, pp. 4809-4814, 1985.

[22] T. Bredow, K. Jug, and R. A. Evarestov, "Electronic and magnetic structure of $\mathrm{ScMnO}_{3}$," Physica Status Solidi, vol. 243, no. 2, pp. R10-R12, 2006.

[23] C. Gatti, V. R. Saunders, and C. Roetti, "Crystal field effects on the topological properties of the electron density in molecular 
crystals: the case of urea," The Journal of Chemical Physics, vol. 101, no. 12, pp. 10686-10696, 1994.

[24] B. H. O'Connor and S. Pratapa, "Improving the accuracy of Rietveld-derived parameters by an order of magnitude," Advances in X-ray Analysis, vol. 45, pp. 158-165, 2002.

[25] W. Marquardt, "An algorithm for least-squares estimation of nonlinear parameters," The SIAM Journal on Applied Mathematics, vol. 11, pp. 431-444, 1963.

[26] F. D. Murnaghan, "The compressibility of media under extreme pressures," Proceedings of the National Academy of Sciences of the United States of America, vol. 30, no. 9, pp. 244-247, 1944.

[27] I. J. Fritz, "Pressure and temperature dependences of the elastic properties of rutile $\left(\mathrm{TiO}_{2}\right)$," Journal of Physics and Chemistry of Solids, vol. 35, no. 7, pp. 817-826, 1974.

[28] R. F. S. Hearmon, "The elastic constants of anisotropic materials," Reviews of Modern Physics, vol. 18, no. 3, pp. 409-440, 1946.

[29] L. Gerward and J. S. Olsen, "Post-rutile high-pressure phases in $\mathrm{TiO}_{2}$," Journal of Applied Crystallography, vol. 30, no. 3, pp. 259-264, 1997.

[30] L. Kóci, D. Y. Kim, J. S. D. Almeida, M. Mattesini, M. M. E. Isaev, and R. Ahuja, "Mechanical stability of $\mathrm{TiO}_{2}$ polymorphs under pressure: $a b$ initio calculations," Journal of Physics Condensed Matter, vol. 20, no. 34, Article ID 345218, 2008.

[31] Z. Jun, Y. J. Xin, W. Y. Ju, C. X. Rong, and J. F. Qian, "First-principles calculations for elastic properties of rutile $\mathrm{TiO}_{2}$ under pressure," Chinese Physics B, vol. 17, no. 6, pp. 2216-2221, 2008.

[32] J. K. Burdett, T. Hughbanks, G. J. Miller, J. W. Richardson, and J. V. Smith, "Structural-electronic relationships in inorganic solids: powder neutron diffraction studies of the rutile and anatase polymorphs of titanium dioxide at 15 and $295 \mathrm{~K}$," Journal of the American Chemical Society, vol. 109, no. 12, pp. 3639-3646, 1987.

[33] S. Ogata, H. Iyetomi, K. Tsuruta et al., "Variable-charge interatomic potentials for molecular-dynamics simulations of $\mathrm{TiO}_{2}$," Journal of Applied Physics, vol. 86, no. 6, pp. 3036-3041, 1999.

[34] K. Held, V. Anisimov, V. Eyert et al., "LDA+DMFT investigations of transition metal oxides and f-electron materials," in Advances in Solid State Physics, vol. 43, pp. 267-286, Springer, Berlin, Germany, 2003. 

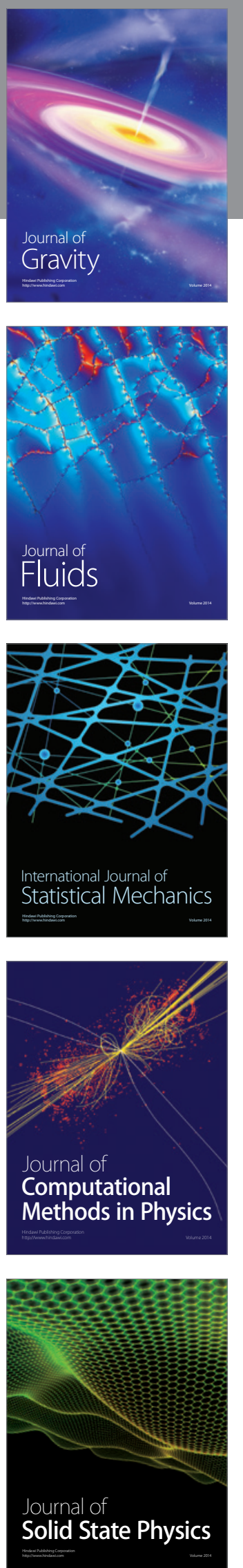

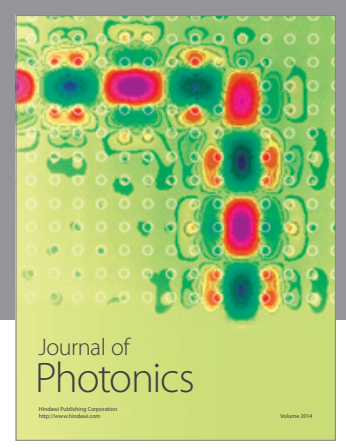

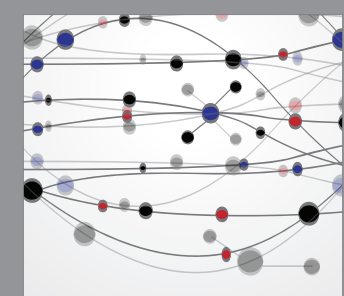

The Scientific World Journal
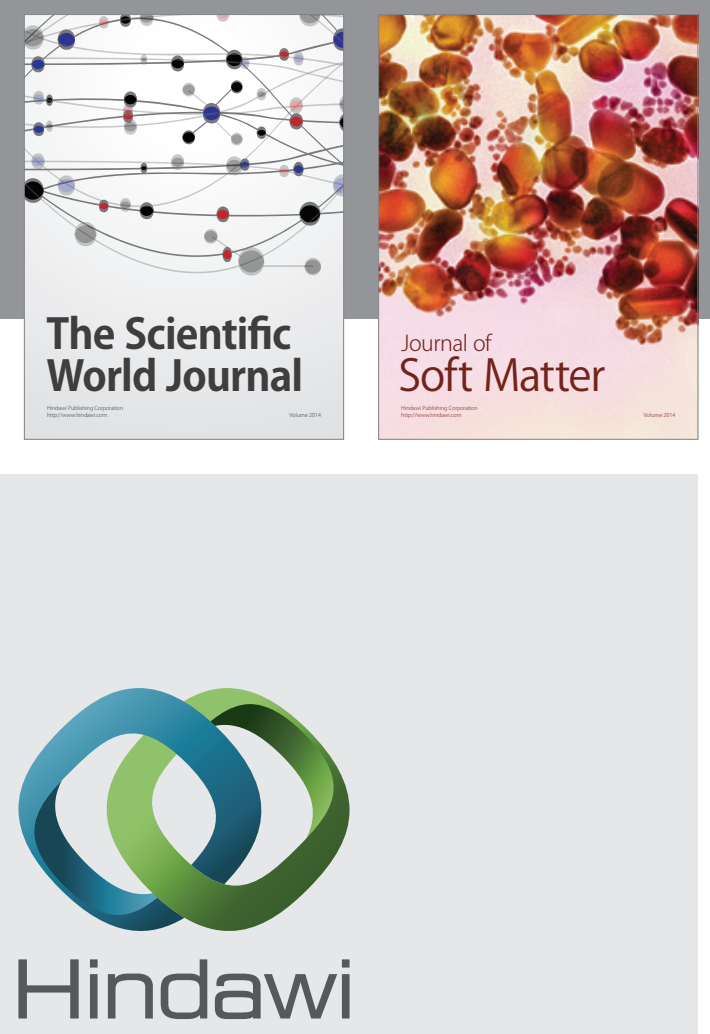

Submit your manuscripts at

http://www.hindawi.com
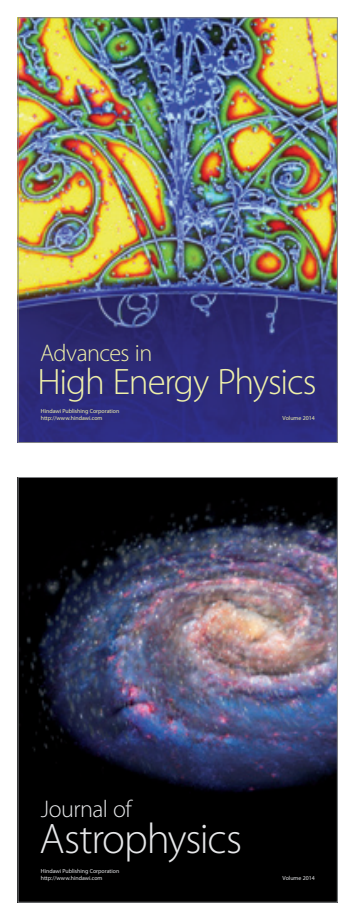
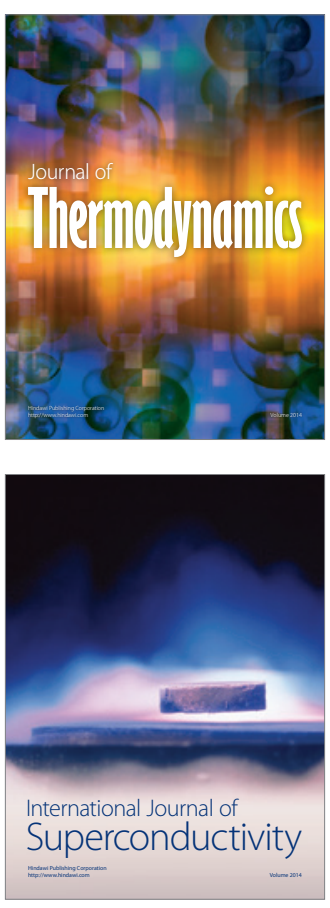
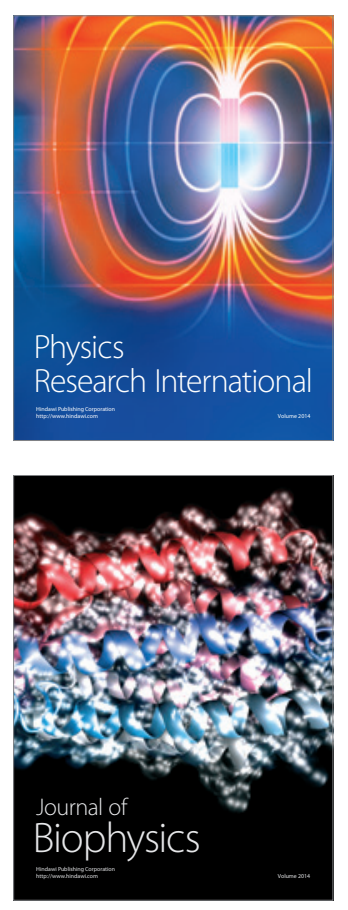
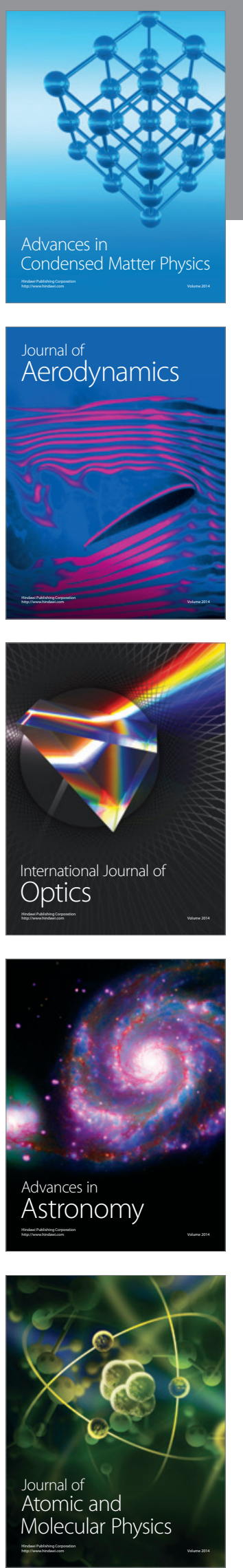